\title{
SPL Marketing Category Terminology
}

National Cancer Institute

\section{Source}

National Cancer Institute. SPL Marketing Category Terminology. NCI Thesaurus. Code C73582.

Terminology used for representation of the information on pharmaceutical product marketing category in the framework of the Structured Product Labeling documents. 